\title{
Comparative All-Cause Mortality Among a Large Population of Patients with Spinal Muscular Atrophy Versus Matched Controls
}

\author{
Emma Viscidi · Maneesh Juneja · Jin Wang • Nasha Wang • \\ Li Li - Wildon Farwell · Ishir Bhan - Corinne Makepeace • \\ Karen Laird · Varant Kupelian · Susan Eaton · Anne Dilley • \\ Susan Hall
}

Received: May 4, 2021 / Accepted: November 23, 2021 / Published online: December 22, 2021

(C) The Author(s) 2021, corrected publication 2022

\section{ABSTRACT}

Introduction: There is little information about survival of spinal muscular atrophy (SMA) patients into adulthood, in particular from population-based samples. We estimated and compared age-specific, all-cause mortality rates in patients with SMA and matched controls in a large, retrospective cohort study using electronic health records (EHRs) from the pretreatment era.

Methods: The US Optum ${ }^{\circledR}$ de-identified EHR database contains EHRs for 104 million persons (study period: January 1, 2007-December $22,2016)$. SMA cases were identified by one or more International Classification of Diseases,

The original version of this article was revised to include the corrected Figure 1.

Supplementary Information The online version contains supplementary material available at https:// doi.org/10.1007/s40120-021-00307-7.

E. Viscidi $(\bowtie) \cdot J$. Wang $\cdot$ N. Wang $\cdot$ L. Li .

W. Farwell · I. Bhan · V. Kupelian · S. Eaton ·

A. Dilley $\cdot$ S. Hall

Biogen, 225 Binney Street, Cambridge, MA 02142,

USA

e-mail: emma.viscidi@biogen.com

M. Juneja

MJ Analytics Ltd, London, UK

C. Makepeace $\cdot$ K. Laird

Biogen, Maidenhead, UK
Ninth/Tenth Edition codes for SMA. Controls with no SMA diagnosis code were matched 10:1 to SMA cases based on birth year, gender, and first diagnostic code date. For both groups, $\geq 1$ month of observation and (if deceased) a valid date of death were required for inclusion. Age-specific mortality rates per person-year (PY) and hazard ratios were calculated. Results: Five thousand one hundred seventynine SMA cases and 51,152 controls were analyzed. The overall hazard ratio comparing cases with controls was 1.76 (95\% CI 1.63-1.90). In patients with SMA type III diagnostic codes only, the all-age mortality rate was 1059/100,000 PYs in cases and 603/100,000 PYs in controls. In older age groups $(13-20,21-30$, $31-40,41-50,51-60$, and $>60$ years), agespecific mortality rates for cases consistently exceeded those of controls. Limitations of this study included the inability to confirm the SMA diagnosis or SMA type by genetic or clinical confirmation.

Conclusion: Patients with SMA of all ages, including adults and type III patients, had a higher all-cause mortality rate as compared to age-matched controls during the pre-treatment era.

Keywords: All-cause mortality; Spinal muscular atrophy; Real-world data; Type III spinal muscular atrophy 


\section{Key Summary Points}

\section{Why carry out this study?}

There is little information about survival of spinal muscular atrophy (SMA) patients into adulthood, and it is commonly accepted that patients with later-onset SMA, who often live to older ages, have a mortality rate similar to the general population.

We estimated and compared age-specific, all-cause mortality rates in patients with SMA and matched controls in a large, retrospective cohort study using electronic health records from the pretreatment era.

\section{What was learned from the study?}

SMA cases had a 1.8-fold higher mortality rate than matched controls.

In patients with SMA type III diagnostic codes only, mortality rates in cases also exceeded controls.

In SMA patients of older ages (13-20, 21-30, 31-40, 41-50, 51-60, and > 60 years), age-specific mortality rates for cases consistently exceeded those of controls.

Patients with SMA, including those with type III SMA and older patients, had a higher all-cause mortality compared with controls during the pre-treatment era.

\section{INTRODUCTION}

Spinal muscular atrophy (SMA) is a neuromuscular disease characterized by the degeneration of motor neurons in the anterior horn of the spinal cord caused by deletions or mutations of the survival motor neuron 1 (SMN1) gene $[1,2]$. The disease results in progressive muscular weakness and can be fatal for untreated patients with the severe phenotype $[1,2]$. Classification is based on age of symptom onset and highest achieved motor function, which correlates with the number of survival motor neuron 2 (SMN2) gene copies [1-3]. Infants diagnosed with SMA type 0 , who have only one $S M N 2$ gene copy, are the most severe; if untreated, they only survive for a few months after birth [3]. Infants with type I make up the majority of incident SMA cases, have symptom onset at age $\leq 6$ months, are typically associated with two SMN2 gene copies, are unable to sit or roll independently, and eventually have difficulty swallowing and require assisted ventilation [1-3]. Children with SMA type II have symptom onset between 6 and 18 months of age, can have 2-4 SMN2 gene copies, and can sit unassisted but may not walk independently [1-3]. Patients with SMA type III have symptom onset after age 18 months and are able to stand and walk unassisted [1, 2]. Patients with SMA type IV have a milder, adultonset form of SMA, and affected individuals can walk independently $[1,2]$.

Despite being a rare disease, SMA was the most common monogenetic cause of death in infants $[4,5]$. Prior to the approval of nusinersen in 2016, untreated SMA type I was associated with early mortality and severe disability in survivors $[1-3,6]$. The approvals of onasemnogene abeparvovec and risdiplam have led to additional treatment options for patients $[7,8]$. Studies have also indicated a shortened life expectancy in children with SMA type II [9-11], whereas it is often reported that the lifespan is normal in patients with SMA type III and IV, who often live to older ages [1, 12, 13]. In prior studies examining survival among patients with SMA in the pre-treatment era, normal survival probabilities were reported for patients with SMA types III and IV when followed up until the age of $\sim 40$ years, but a decline in survival was observed in SMA type II [14]. Earlier reports of mortality among patients with SMA were from smaller, specialist centers; studies of survival of patients with SMA in larger and more diverse medical settings are rare [15]. Furthermore, most prior studies examined survival only in patients with SMA and did not perform a comparison with healthy controls [14]. 
In this study, we estimated and compared the age-specific, all-cause mortality rates in patients with SMA and matched control patients in a large, retrospective cohort study based on US electronic health records (EHRs) collected prior to the approval of nusinersen.

\section{METHODS}

Patients were identified from the US Optum ${ }^{\circledR}$ EHR de-identified database, which contains EHRs for $\sim 104$ million persons. The database contains extensive clinical encounter data from a network of more than 140,000 providers at more than 700 hospitals and more than 7000 clinics across the US. The database encapsulates all demographics and US census divisions and regions, and includes patients with all types of insurance in the US, both private and public. Patients are enrolled based on their insurance coverage and, therefore, patients may enter or exit the database at different time points depending on their insurance coverage. The study period was January 1, 2007-December 22, 2016 (the time period prior to the approval of nusinersen in the United States). The case definition of SMA was a patient having one or more International Classification of Diseases, Ninth and/or Tenth Edition (ICD-9/ICD-10) codes for SMA appearing as primary, admission, or discharge diagnoses $(335.0,335.10,335.11$, 335.19, G12.0, G12.1, G12.8, and G12.9) (Supplementary Table SI). Included diagnostic codes for SMA were specific to Werdnig-Hoffmann disease (SMA type I), Kugelberg-Welander disease (SMA type III), and unspecified SMA. The date of the first diagnostic code was defined as the date of the first recorded SMA code in the database. A stratified analysis of patients with diagnosis codes for type III SMA only was conducted (defined as patients with one or more ICD-9 code 335.11 and no other SMA diagnostic codes).

Females with a record of a pregnancy (based on ICD-9 and ICD-10 diagnostic codes) in the 1-year time period before or after the SMA first diagnostic code date were excluded from the case definition. This was done to reduce the risk of erroneously classifying women with babies that were diagnosed with SMA as SMA cases themselves (Supplementary Table SII).

Controls with no SMA diagnosis code at any time in the history of the database were matched 10:1 to SMA cases based on birth year, gender, and the date of the first diagnosis code for the SMA cases (for the purposes of a matched longitudinal follow-up). For both groups, $\geq 1$ month of observation and (if deceased) a valid date of death were required for inclusion. All study participants were followed for death due to any cause. Age-specific, allcause mortality rates per 100,000 person-years (PYs), 95\% confidence intervals (CIs), and hazard ratios (HRs) were calculated among cases versus controls using SAS software (SAS Institute Inc., Cary, NC, USA). As a comparison with the general population, age-specific, all-cause mortality rates at the midpoint of the study period (2012) were also calculated for the US population based on data from the Centers for Disease Control and Prevention WONDER Online Database [16]. Data for these analyses were made available to the authors through a thirdparty license from Optum, a commercial data provider in the US. Ethics committee approval was not required for this study due to the use of secondary de-identified data.

\section{RESULTS}

A total of 5179 patients who met the SMA case definition were identified and matched to 51,152 controls. Approximately $12 \%$ of patients with SMA were age $<13$ years at the first SMA diagnostic code date, and $31 \%$ were age $>60$ years (Table 1$)$. Over half $(53 \%)$ of SMA cases were male, $80 \%$ were non-Hispanic, and $77 \%$ were Caucasian. SMA cases and controls had a mean (standard deviation) of 7.7 (3.8) years of observation time from the first diagnostic code date. A total of $746(14 \%)$ patients with SMA died during the follow-up period, compared with 4227 (8.3\%) deaths in the control group. There were 66 patients with type III SMA only (Table 1). Among those patients, the mean age was 38.9 years and approximately half were female. 
Table 1 Analysis sample characteristics of all SMA cases, type III SMA cases, and matched controls

\begin{tabular}{|c|c|c|c|}
\hline & $\begin{array}{l}\text { All SMA } \\
\text { cases } \\
(N=5179)\end{array}$ & $\begin{array}{l}\text { Matched } \\
\text { controls } \\
(N=51,152)\end{array}$ & $\begin{array}{l}\text { Type III } \\
\text { SMA cases } \\
(N=66)\end{array}$ \\
\hline \multicolumn{4}{|c|}{ Age at first diagnostic code date, years } \\
\hline $\begin{array}{r}\text { Mean } \\
(\mathrm{SD})\end{array}$ & $45.1(23.5)$ & $45(23.4)$ & $38.9(21.8)$ \\
\hline Median & 45 & 45 & 38 \\
\hline Range & $0-86$ & $0-86$ & $2-82$ \\
\hline \multicolumn{4}{|c|}{ Age at first diagnostic code date, years (\%) } \\
\hline$<1$ & $86(1.7)$ & $859(1.7)$ & $0(0.0)$ \\
\hline 1 to $<2$ & $104(2.0)$ & $1040(2.0)$ & $0(0.0)$ \\
\hline 2 to $<13$ & $427(8.2)$ & $4179(8.2)$ & $9(14.0)$ \\
\hline $13-20$ & $313(6.0)$ & $3087(6.0)$ & $9(14.0)$ \\
\hline $21-30$ & $420(8.1)$ & $4189(8.2)$ & $8(12.0)$ \\
\hline $31-40$ & $910(17.6)$ & $9063(17.7)$ & $10(15.0)$ \\
\hline $41-50$ & $639(12.3)$ & $6348(12.4)$ & $7(11.0)$ \\
\hline $51-60$ & $688(13.0)$ & $6824(13.0)$ & $13(20.0)$ \\
\hline$>60$ & $1592(31.0)$ & $15,563(30.0)$ & $10(15.0)$ \\
\hline \multicolumn{4}{|c|}{ Gender, $n(\%)$} \\
\hline Female & $2457(47.4)$ & $24,324(47.6)$ & $32(48.0)$ \\
\hline Male & $2722(52.6)$ & $26,828(52.4)$ & $34(52.0)$ \\
\hline \multicolumn{4}{|c|}{ Ethnicity, $n$ (\%) } \\
\hline Hispanic & $263(5.1)$ & $2937(5.7)$ & $0(0.0)$ \\
\hline $\begin{array}{l}\text { Non- } \\
\text { Hispanic }\end{array}$ & $4167(80.5)$ & $39,601(77.4)$ & $53(80.0)$ \\
\hline Unknown & $749(14.5)$ & $8614(16.8)$ & $13(20.0)$ \\
\hline \multicolumn{4}{|l|}{ Race, $n(\%)$} \\
\hline Asian & $177(3.4)$ & $1056(2.1)$ & $0(0.0)$ \\
\hline $\begin{array}{l}\text { Black/ } \\
\text { African } \\
\text { American }\end{array}$ & $374(7.2)$ & $4840(9.5)$ & $6(9.1)$ \\
\hline White & $3967(76.6)$ & $37,171(72.7)$ & $51(77.0)$ \\
\hline $\begin{array}{l}\text { Other/ } \\
\text { unknown }\end{array}$ & $661(12.8)$ & $8085(15.8)$ & $9(14.0)$ \\
\hline
\end{tabular}

Table 1 continued

\begin{tabular}{|c|c|c|}
\hline 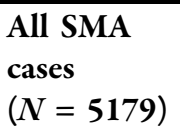 & 1 & \\
\hline
\end{tabular}

Time in database, years

$\begin{array}{llll}\begin{array}{l}\text { Mean } \\ (\mathrm{SD})\end{array} & 7.7(3.8) & 7.7(3.8) & 8.9(3.6) \\ \text { Median } & 7.8 & 7.8 & 10.0 \\ \text { Range } & 0.2-13.8 & 0.2-13.8 & 0.6-13.7\end{array}$

Died during follow-up, $n$ (\%)

\begin{tabular}{llll} 
No & $4433(85.6)$ & $46,925(91.7)$ & $60(91.0)$ \\
Yes & $746(14.4)$ & $4227(8.3)$ & $6(9.1)$ \\
\hline
\end{tabular}

First diagnostic code date in patients with SMA was defined as the date of the first recorded SMA code. Controls were matched 10:1 to SMA cases based on birth year, gender, and first diagnostic code date. Type III SMA cases are patients with diagnostic codes for ICD-9 335.11 only $S D$ standard deviation, $S M A$ spinal muscular atrophy

The overall all-cause mortality rate was 1926 per 100,000 PYs (95\% CI 1792-2069) for SMA cases and 1099 per 100,000 PYs (95\% CI 1066-1132) for controls (Fig. 1; Table 2). The HR comparing SMA cases with matched controls was 1.76 (95\% CI 1.63-1.90). Among females with SMA, the all-cause mortality rate was 1786 per 100,000 PYs (95\% CI 1606-1987) and 970 per 100,000 PYs (95\% CI 926-1015) among female controls. The mortality rate was also higher in males with SMA than in male controls (2061 vs. 1223 per 100,000 PYs) (Table 2). The genderspecific HR comparing SMA cases with controls was 1.85 (95\% CI 1.65-2.08) and 1.69 (95\% CI 1.52-1.88) for females and males, respectively.

Every age-specific mortality rate in SMA cases exceeded that in controls, and the HRs comparing SMA cases with controls were statistically significant in all age groups (Fig. 1; Table 2). The largest HR was observed in the $<1$ year age group [HR 43.96 (95\% CI 19.25-100.36)], which is not surprising given that these were mostly patients with SMA type I, who have poor survival when untreated. In older patients, every age-specific mortality rate $(13-20,21-30,31-40$, $41-50,51-60$, and $>60$ years) in SMA cases 


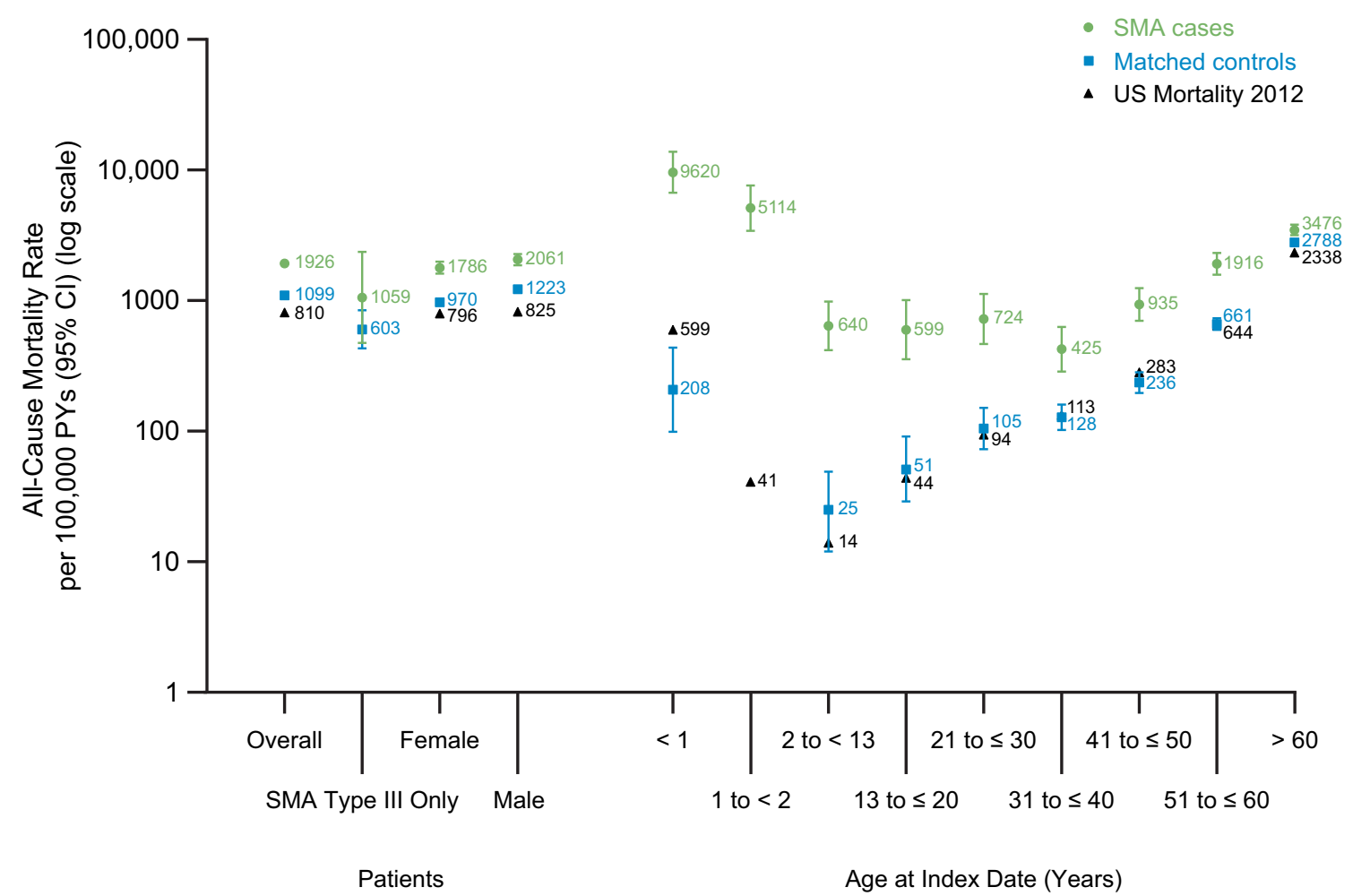

Fig. 1 All-cause mortality rate per 100,000 PYs among SMA cases, matched controls, and the US general population overall, for SMA Type III only, and by gender and age at first diagnostic code date (grouped). See Table 2 for the $95 \%$ CIs for the US general population, which are

exceeded the rate observed in controls, with HRs ranging between 1.26 and 11.66. Among patients in the oldest age group ( $>60$ years), mortality in SMA cases was 3476 per 100,000 PYs, as compared to 2788 per 100,000 PYs in controls [HR 1.26 (95\% CI 1.14-1.39)].

In patients with SMA type III only, the allage mortality rate was $1059 / 100,000$ PYs in cases and 603/100,000 PYs in controls [HR 1.76 (0.74-4.20)].

All-cause mortality in the US population compared with SMA cases and controls is shown in Fig. 1 and Table 2. The overall mortality rate in SMA cases (1926 per 100,000 PYs) was twofold higher than that in the US population (810 per 100,000 PYs); the rate among the study controls (1099 per 100,000 PYs) was also higher than-but comparatively more similar to-that in the US population. not visible in the figure. First diagnostic code date in patients with SMA was defined as the date of the first recorded SMA code. US mortality in 2012 is from the CDC WONDER Online Database [16]. CI confidence interval, $P Y$ person-year, $S M A$ spinal muscular atrophy

\section{DISCUSSION}

This large US database study, conducted during the pre-treatment era, found that all-cause mortality was twofold higher among SMA cases compared with their matched non-SMA controls. The higher mortality rate was observed in every age group examined, including older age groups ( $>13$ years), as well as in patients with SMA type III diagnostic codes only (although the hazard ratio in this group did not reach statistical significance). This finding suggests that, in the pre-treatment era, SMA patients of all ages have a higher mortality rate than the general population, including older patients who survive into adulthood and those with SMA type III $[1,10,11,13]$. This is one of the first studies to examine mortality in patients with SMA compared with a representative 
Table 2 All-cause mortality rate per 100,000 PYs among SMA cases, matched controls, and the US general population overall, for SMA type III only, and by gender and age at first diagnostic code date

\begin{tabular}{lllll}
\hline & $\begin{array}{l}\text { SMA cases } \\
(\boldsymbol{N}=\mathbf{5 1 7 9 )} \\
\text { Rate }(\mathbf{9 5 \%} \mathbf{C I})\end{array}$ & $\begin{array}{l}\text { Matched controls } \\
(\boldsymbol{N}=\mathbf{5 1 , 1 5 2}) \\
\text { Rate }(\mathbf{9 5 \%} \mathbf{C I})\end{array}$ & $\begin{array}{l}\text { HR comparing SMA cases to } \\
\text { controls } \\
\text { HR }(\mathbf{9 5 \%} \mathbf{C I})\end{array}$ & $\begin{array}{l}\text { US mortality in } \\
\mathbf{2 0 1 2}[\mathbf{1 6}] \\
\text { Rate (95\% CI) }\end{array}$ \\
\hline $\begin{array}{l}\text { Overall } \\
\text { SMA type III } \\
\text { only }\end{array}$ & $1926(1792-2069)$ & $1099(1066-1132)$ & $1.76(1.63-1.90)$ & $810(809-811)$ \\
Female & $1786(1606-1987)$ & $970(926-1015)$ & $1.85(1.65-2.08)$ & NA \\
Male & $2061(1870-2271)$ & $1223(1175-1273)$ & $1.69(1.52-1.88)$ & $796(795-798)$ \\
Age at first diagnostic code date, years & & & $825(823-826)$ \\
$<1$ & $9620(6685-13,843)$ & $208(99-437)$ & $43.96(19.25-100.36)$ & $599(592-607)$ \\
1 to $<2$ & $5114(3428-7630)$ & $0^{\mathrm{b}}$ & - & $41(39-43)$ \\
2 to $<13$ & $640(418-982)$ & $25(12-49)$ & $26.0(11.52-58.70)$ & $14(14-15)$ \\
13 to $\leq 20$ & $599(355-1011)$ & $51(29-91)$ & $11.66(5.39-25.20)$ & $44(43-44)$ \\
21 to $\leq 30$ & $724(467-1123)$ & $105(73-151)$ & $6.89(3.90-12.18)$ & $94(94-95)$ \\
31 to $\leq 40$ & $425(287-630)$ & $128(102-160)$ & $3.33(2.12-5.23)$ & $133(132-134)$ \\
41 to $\leq 50$ & $935(701-1249)$ & $236(196-283)$ & $3.99(2.83-5.61)$ & $283(282-285)$ \\
51 to $\leq 60$ & $1916(1584-2318)$ & $661(597-732)$ & $2.90(2.34-3.61)$ & $644(641 / 6-646)$ \\
$>60$ & $3476(3173-3808)$ & $2788(2699-2881)$ & $1.26(1.14-1.39)$ & $2338(2334-2343)$ \\
\hline
\end{tabular}

First diagnostic code date in patients with SMA was defined as the date of the first recorded SMA code. US mortality in 2012 was taken from the CDC WONDER Online Database [16]

$C I$ confidence interval, $H R$ hazard ratio, $N A$ not applicable, $P Y$ person-year, SMA spinal muscular atrophy, Type III SMA only are patients with SMA diagnostic codes for ICD-9 335.11 only (i.e., no other SMA diagnostic codes)

${ }^{a} N=66$ SMA type III only cases and $N=631$ matched controls

${ }^{\mathrm{b}}$ There were no deaths among the 1040 controls aged 1 to $<2$ years

sample of age-matched controls in a large, realworld sample. The current study examined over 5000 SMA patients, including 1592 patients over 60 years of age. Prior studies of SMA mortality had smaller sample sizes of $<150$ patients and many have only followed patients up to $\sim 60$ years of age [14].

In a prior matched cohort study using US claims data conducted in the pre-SMA treatment era, 296 patients with later-onset SMA (defined as a first diagnosis of SMA after 3 years of age) were matched to controls without SMA over a similar 10-year study timeframe (January 2006-March 2016) [17]. The mortality rate in the later-onset SMA cohort was 5.4\% compared with $2.3 \%$ in controls. The average time from index date (defined as the date of the first SMArelated diagnosis code) to death was $26.2 \pm 19.75$ and $36.4 \pm 23.13$ months in the later-onset SMA cohort and non-SMA cohort, respectively. Although the mortality rate was higher in SMA cases, no statistically significant differences in mortality risk were found between the two groups based on a KaplanMeier analysis [17]. In contrast, the present study observed a statistically significant increase in mortality among SMA cases compared with controls, likely due to the larger sample size of this population-based study, which included 10 matched controls for every SMA case. The 
present study also compared mortality in patients with SMA to the mortality rate in the general population.

In a recent study of survival in a populationbased cohort of 307 treatment-naive patients with SMA in the Netherlands, patients with SMA types I-IV were enrolled between September 2010 and August 2014 [14]. Shortened survival was observed in SMA types I and IIa, but not in types IIb, III, and IV [14]. The majority of patients with SMA type I require mechanical ventilation from an early age and typically die from respiratory failure, while patients with SMA types II-III vary in their use of ventilatory support $[9,14]$. The use of noninvasive ventilatory support has been available to SMA patients since the early 1990s and most likely did not have an impact on the survival scores in this cohort, since the study period started in 2007 [18]. The data indicated close-to-normal survival probabilities for SMA types IIb, III, and IV within the first 50-60 years of life but did not follow patients beyond that age [14]. The present study examined a larger sample of SMA patients, including over 4000 adults aged 21 years and older.

Mortality rates among controls in our sample were similar to-but slightly higher thanthose in the general US population, as reported by the Centers for Disease Control and Prevention [16]. The higher mortality in the control group likely reflects differences in the age and gender distribution of controls compared with the US population (for example, 53\% of SMA cases and controls were males, while the gender distribution was slightly different (49\% were males) in the 2010 census data for the general US population) [19]. Furthermore, the controls in this study were derived from an EHR database; patients in the database may be more likely to receive medical care and may require more medical care than those in the general population.

The limitations of this analysis include the use of diagnostic codes to define SMA, a lack of information on the specific SMA phenotype/ SMA type, and the lack of information on the age at first diagnosis of SMA. In this study we were unable to confirm the SMA diagnosis by genetic or clinical confirmation. There is no validated diagnostic algorithm for using codes for SMA in databases; however, we used SMAspecific ICD codes to ensure that those with other neuromuscular disorders were not captured. In addition, we examined patients with SMA type III diagnostic codes only to examine this subgroup of SMA patients. Prior studies have used a similar methodology to identify SMA patients from healthcare records [20]. As such, there is the possibility of misclassification of cases in the SMA group. We did not evaluate individual causes of death in this study, but this topic could be addressed in future studies.

The strengths of this study include the use of a large real-world sample of patients with SMA from diverse medical care settings, which may have less selection bias than previous, smaller studies of patients from specialist centers. While the Optum database may not be representative of the entire US population, SMA patients in this database represent a wider range of patients than those in samples from specialist centers or single medical institutions because they are patients receiving care from healthcare facilities across the nation and patients with different types of health insurance. In this study, SMA cases were matched to a large population of controls, providing sufficient power to observe statistically significant differences. The cohort in this study included SMA cases with a wide range of ages at first diagnostic code date, allowing for the evaluation of both adult and pediatric SMA patients. This study also utilized an EHR database, which provides a more comprehensive profile of patient health records than administrative claims data.

\section{CONCLUSIONS}

The findings of this study indicate that the SMA disease state, even for patients surviving to older ages and those who have SMA type III, is associated with decreased life expectancy in the pre-treatment era. In the future, it is recommended that mortality studies of patients with SMA include a detailed analysis of cause of death and genetic confirmation of SMA, which will provide detailed mortality rates in clinical studies and elucidate the potential reason for 
higher mortality in SMA patients. Our results suggest the need for improvements in the care and management of patients with SMA, regardless of age or SMA type.

\section{ACKNOWLEDGEMENTS}

Funding. This study was supported by Biogen (Cambridge, MA, USA). The study sponsor is also funding the journal's Rapid Service Fee.

Medical Writing, Editorial, and Other Assistance. Biogen provided funding for medical writing support in the development of this paper; Nancy Niguidula of Excel Scientific Solutions (Fairfield, CT, USA) provided medical writing support, and Nathaniel Hoover from Excel Scientific Solutions copyedited and styled the manuscript per journal requirements. Critical review was provided by Jim Gurr, PhD, Julie Welch, PhD, Angela D. Paradis, ScD, and Christine Lee, PhD, JD of Biogen, Cambridge, MA, USA.

Authorship. All named authors meet the International Committee of Medical Journal Editors (ICMJE) criteria for authorship for this article, take responsibility for the integrity of the work as a whole, and have given their approval for this version to be published.

Authors' Contributions. Emma Viscidi: concept and design, statistical analysis, drafting the manuscript; Maneesh Juneja: concept and design, statistical analysis; Jin Wang: statistical analysis; Nasha Wang: concept and design, statistical analysis; Li Li: statistical analysis; Wildon Farwell: concept and design, drafting the manuscript; Ishir Bhan: concept and design, drafting the manuscript; Corinne Makepeace: concept and design, drafting the manuscript; Karen Laird: concept and design, drafting the manuscript; Varant Kupelian: concept and design, statistical analysis, drafting the manuscript; Susan Eaton: concept and design, statistical analysis; Anne Dilley: concept and design, drafting the manuscript; Susan Hall: concept and design, statistical analysis, drafting the manuscript.

Disclosures. Emma Viscidi, Jin Wang, Nasha Wang, Li Li, Corinne Makepeace, Karen Laird, Varant Kupelian, and Susan Eaton are employees of and hold stock/stock options in Biogen. Maneesh Juneja is an employee of MJ Analytics Ltd. Wildon Farwell, Ishir Bhan, Anne Dilley, and Susan Hall are former employees of and hold stock/stock options in Biogen. Wildon Farwell is an employee of Dyne Therapeutics Waltham, MA; Anne Dilley is an independent contractor; Susan Hall is an employee of Moderna Cambridge, MA.

Compliance with Ethics Guidelines. Data for these analyses were made available to the authors through third-party license from Optum, a commercial data provider in the United States. Ethics committee approval was not required for this study due to the use of secondary, de-identified data.

Data Availability. The dataset supporting the conclusions of this article are available in the US Optum ${ }^{\circledR}$ EHR repository and are hyperlinked to dataset(s) in https://www.optum.com/ business/solutions/government/federal/dataanalytics-federal/clinical-data.html\#.

Open Access. This article is licensed under a Creative Commons Attribution-NonCommercial 4.0 International License, which permits any non-commercial use, sharing, adaptation, distribution and reproduction in any medium or format, as long as you give appropriate credit to the original author(s) and the source, provide a link to the Creative Commons licence, and indicate if changes were made. The images or other third party material in this article are included in the article's Creative Commons licence, unless indicated otherwise in a credit line to the material. If material is not included in the article's Creative Commons licence and your intended use is not permitted by statutory regulation or exceeds the permitted use, you will need to obtain permission directly from the copyright holder. To view a copy of this licence, 
visit http://creativecommons.org/licenses/by$\mathrm{nc} / 4.0 /$.

\section{REFERENCES}

1. Markowitz JA, Singh P, Darras BT. Spinal muscular atrophy: a clinical and research update. Pediatr Neurol. 2012;46(1):1-12.

2. Darras BT, Monani UR, De Vivo DC, et al. Genetic disorders affecting the motor neuron: spinal muscular atrophy. In: Swaiman KF, Ashwal S, Ferriero DM, et al., editors. Swaiman's pediatric neurology: principles and practice. 6 th ed. Edinburgh: Elsevier; 2017. p. 1057-64.

3. Mercuri E, Bertini E, Iannaccone ST. Childhood spinal muscular atrophy: controversies and challenges. Lancet Neurol. 2012;11(5):443-52.

4. Sugarman EA, Nagan N, Zhu H, et al. Pan-ethnic carrier screening and prenatal diagnosis for spinal muscular atrophy: clinical laboratory analysis of $>72,400$ specimens. Eur J Hum Genet. 2012;20(1): 27-32.

5. Miniño AM, Xu J, Kochanek KD. Deaths: preliminary data for 2008. Natl Vital Stat Rep. 2010;59(2): $1-52$.

6. Biogen Inc. Package insert for SPINRAZA (nusinersen). Cambridge, MA: Biogen Inc.; 2016.

7. Genentech, Inc. Package insert for EVRYSDI (risdiplam). South San Francisco, CA: Genentech, Inc.; 2020.

8. AveXis, Inc. Package insert for ZOLGENSMA (onasemnogene abeparvovec-xioi). Bannockburn, IL: AveXis, Inc.; 2019.

9. Farrar MA, Vucic S, Johnston HM, du Sart D, Kiernan MC. Pathophysiological insights derived by natural history and motor function of spinal muscular atrophy. J Pediatr. 2013;162(1):155-9.
10. Zerres K, Rudnik-Schöneborn S, Forrest E, Lusakowska A, Borkowska J, Hausmanowa-Petrusewicz I. A collaborative study on the natural history of childhood and juvenile onset proximal spinal muscular atrophy (type II and III SMA): 569 patients. J Neurol Sci. 1997;146(1):67-72.

11. Arnold WD, Kassar D, Kissel JT. Spinal muscular atrophy: diagnosis and management in a new therapeutic era. Muscle Nerve. 2015;51(2):157-67.

12. Moulard B, Salachas F, Chassande B, et al. Association between centromeric deletions of the SMN gene and sporadic adult-onset lower motor neuron disease. Ann Neurol. 1998;43(5):640-4.

13. Russman BS. Spinal muscular atrophy: clinical classification and disease heterogeneity. J Child Neurol. 2007;22(8):946-51.

14. Wijngaarde CA, Stam M, Otto LAM, et al. Population-based analysis of survival in spinal muscular atrophy. Neurology. 2020;94(15):e1634-44.

15. Kolb SJ, Coffey CS, Yankey JW, et al. Natural history of infantile-onset spinal muscular atrophy. Ann Neurol. 2017;82(6):883-91.

16. Centers for Disease Control and Prevention. CDC WONDER. https://wonder.cdc.gov/. Accessed 4 Jan 2019.

17. Tan H, Gu T, Chen E, Punekar R, Shieh PB. Healthcare utilization, costs of care, and mortality among patients with spinal muscular atrophy. J Health Econ Outcomes Res. 2019;6(3):185-95.

18. Oskoui M, Levy G, Garland CJ, et al. The changing natural history of spinal muscular atrophy type 1 . Neurology. 2007;69(20):1931.

19. US Census Bureau. Age and sex composition: 2010. https://www.census.gov/prod/cen2010/briefs/ c2010br-03.pdf. Accessed Apr 2021.

20. Lipnick SL, Agniel DM, Aggarwal R, et al. Systemic nature of spinal muscular atrophy revealed by studying insurance claims. PLoS One. 2019;14(3): e0213680. 